\title{
Response Predicting LTCC Firing Shrinkage: A Response Surface Analysis Study
}

\author{
Michael Girardi, Gregg Barner, Cristie Lopez, Brent Duncan, Larry Zawicki \\ Honeywell Federal Manufacturing \& Technologies*
}

\begin{abstract}
The Low Temperature Cofired Ceramic (LTCC) technology is used in a variety of applications including military/space electronics, wireless communication, MEMS, medical and automotive electronics. The use of LTCC is growing due to the low cost of investment, short development time, good electrical and mechanical properties, high reliability, and flexibility in design integration (3 dimensional (3D) microstructures with cavities are possible)). The dimensional accuracy of the resulting $x / y$ shrinkage of LTCC substrates is responsible for component assembly problems with the tolerance effect that increases in relation to the substrate size. Response Surface Analysis was used to predict product shrinkage based on specific process inputs (metal loading, layer count, lamination pressure, and tape thickness) with the ultimate goal to optimize manufacturing outputs (NC files, stencils, and screens) in achieving the final product design the first time. Three (3) regression models were developed for the DuPont 951 tape system with DuPont 5734 gold metallization based on green tape thickness.
\end{abstract}

Keywords: LTCC, Design of Experiment, shrinkage modeling

*Operated for the United States Department of Energy under Contract No. DE-ACO4-01AL66850

\section{Background:}

Low Temperature Cofired Ceramic (LTCC) technology incorporates high density ceramic packaging and low temperature processing. Many passive components can be buried into a multi-layer, monolithic structure and fired simultaneously [1].

LTCC processing begins with a ceramic tape. The tape consists of a glass and filler dielectric material suspended in organic binders and plasticizers. To create the tape, a wet mixture called a slurry, is cast onto a backing material using a continuous tape caster. The tape is cast at uniform thickness, typically between 2-10 mils, and then immediately dried. After the tape is created, it is rolled up and stored. Commercial LTCC tape can be purchased either in these rolls or pre-cut to a specific size.

The following explains the researchers' LTCC process flow:

After the tape is blanked to the correct size, it is placed in an oven for 1 hour at $100^{\circ} \mathrm{C}$. This conditioning process replaces the previous practice of normalization, i.e. removing the backing material and allowing the tape to settle for 24 hours. The benefit of conditioning rather than normalization is that the backing material does not have to be removed from the tape. This keeps the tape rigid during the single-layer processing steps. The benefit of tape rigidity is to restrict tape movement (stretching can cause misalignments and compromise shrinkage measurements at the surface).

The conditioned tape is then punched. Via holes and registration holes are punched at the same time to maximize alignment between layers.

The punched tape is then screen printed. The vias are filled first, using a very dense material that does not fall out during firing. After all vias are filled and dried, conductor traces are printed on the exposed tape surface, or front side. On layers requiring backside printing, the backing material is removed to allow printing.

After the conductor prints are dried, the backing material is removed, and the layers are stacked using a mechanical fixture with pins. Each stack is then wrapped in latex, vacuum sealed in bags, and laminated. The laminated panels are then baked out and fired in a box furnace.

Standard, unconstrained LTCC firing shrinkage ranges from $10-15+\%$ in the $\mathrm{X}$ and $\mathrm{Y}$ directions to $20 \%$ in the $\mathrm{Z}$ direction [2], depending on the 
tape. LTCC metallizations are designed to perform similarly to the LTCC tapes during firing. LTCC panels should not show excessive warping in the areas that are printed. However, as LTCC designs become more complex and require increased amounts of metallization, the overall $\mathrm{X}-\mathrm{Y}$ shrinkage of the panel decreases because of high metallization density. This has created many difficulties, not only in post-fired printing, but also in assembly operations. In wire bonding and die place, where most commercial operations are done automatically, a significant variation in sizing from the original design can have a catastrophic effect on yield.

Determining optimal lamination and firing parameters for each design can cause many delays. Designers historically used a specific expansion factor for every build based on an average of past data, and artwork for all cofired metallization screens and stencils were created using this factor. Screens for post-fire printing were made assuming 1:1 correlation with the cofired artwork expansion factor once the panels are fired. After firing, if the actual shrinkage amount did not match the predicted value, a lamination study was completed to determine the optimal lamination pressure to achieve the desired shrinkage. This would take anywhere from two days to a week to complete, and consumed product as well as labor. At times, the shrinkage was so low that the designer would have to modify the artwork using the actual expansion factor for the particular build, and in turn new screens would be made for post-fired printing increasing costs and delaying production.

In order to save labor, material costs, and time in the fabrication process, it was decided to perform a DOE and regression analysis to predict a shrinkage value for each design using a formula rather than trial and error. With the changing layer count, metal loading and tape layer requirements of various designs, it was determined that a shrinkage model was needed to better center the lamination process (3000 psi target).

\section{Experimental Design:}

Following screening experiments used to select main factors and test levels, a Central Composite 20 run, 3 factor, with 8 cube points, 4 center points on cube, 6 axial points and 2 center points in axial DOE was completed on DuPont 951 LTCC tape. The shrinkage behavior was modeled separately on three LTCC green tape thicknesses as follows: 4.5, 6.5, and 10 mil thicknesses. The input factors and levels consisted of the following:

$\begin{array}{lrr} & \text { Low } & \text { High } \\ \text { Metal Loading } & 20 \% & 80 \% \\ \text { Lamination Pressure } & 2750 \mathrm{psi} & 3100 \mathrm{psi} \\ \text { Layer Count } & 7 & 17\end{array}$

The constants for the DOE were as follows: the same tape lot for a given thickness, the samples were fired at $850^{\circ} \mathrm{C}$ in the same box oven, $8 \mathrm{um}$ fired thickness using DuPont 5734 cofire Au was targeted, cavities were not present, and lamination of the green tape occurred in an isostatic laminator at a temperature of $70^{\circ} \mathrm{C}$ for 10 minutes after 5 minutes of preheat. The metal loading was determined by layer from gerber files generated by CAM360 V9.5 using the Copper Analysis tool.

The response factor for the DOE was the average shrinkage based on a sample size of one 5" square panel (due to cost constraints). Green and fired punched holes in the LTCC tape were measured diagonally on each sample using an optical measurement system.

\section{DuPont 10 Mil Thick 951 PX Green Tape}

The DOE run combinations and results for the 10 mil thick green tape following randomization can be broken down as shown in Table 1.

Table 1

\begin{tabular}{|cccccc}
\hline Run Order & metal loading & pressure & layer count & shrinkage \\
1 & 25 & 3200 & 17 & 12.51 \\
2 & 50 & 3000 & 12 & 12.33 \\
3 & 50 & 3000 & 12 & 12.34 \\
4 & 50 & 3000 & 12 & 12.29 \\
5 & 25 & 2800 & 17 & 12.65 \\
6 & 75 & 3200 & 7 & 12.30 \\
7 & 50 & 3000 & 12 & 12.32 \\
8 & 25 & 2800 & 7 & 12.56 \\
9 & 75 & 3200 & 17 & 12.24 \\
10 & 25 & 3200 & 7 & 12.44 \\
11 & 75 & 2800 & 17 & 12.41 \\
12 & 75 & 2800 & 7 & 12.38 \\
13 & 50 & 2673 & 12 & 12.41 \\
14 & 0 & 3000 & 12 & 12.73 \\
15 & 50 & 3000 & 12 & 12.33 \\
16 & 50 & 3000 & 12 & 12.30 \\
17 & 50 & 3327 & 12 & 12.18 \\
18 & 50 & 3000 & 4 & 12.31 \\
19 & 100 & 3000 & 12 & 12.29 \\
20 & 50 & 3000 & 20 & 12.34 \\
\hline
\end{tabular}

The analysis of variance (ANOVA), calculated by Minitab v15 can be summarized as shown in Table 2. 
Table 2

Response Surface Regression: shrinkage versus Block, metal loading, pressure

Estimated Regression Coefficients for shrinkage PX

$\begin{array}{lrrrc}\text { Term } & \text { Coef } & \text { SE Coef } & \text { T } & \text { P } \\ \text { Constant } & 12.3794 & 0.01930 & 641.279 & 0.000 \\ \text { Block } & 0.0181 & 0.01930 & 0.939 & 0.363 \\ \text { metal loading } & -0.1325 & 0.02622 & -5.054 & 0.000 \\ \text { pressure } & -0.0664 & 0.02316 & -2.867 & 0.012 \\ \text { metal*pressure } & 0.0016 & 0.03708 & 0.042 & 0.967\end{array}$

$\mathrm{S}=0.08459 \quad \mathrm{R}-\mathrm{Sq}=69.8 \% \mathrm{R}-\mathrm{Sq}(\mathrm{adj})=61.7 \%$

Estimated Regression Coefficients for shrinkage PX using data in uncoded units

$\begin{array}{ll}\text { Term } & \text { Coef } \\ \text { Constant } & 13.6269 \\ \text { Block } & 0.0181250 \\ \text { metal loading } & -0.00502500 \\ \text { pressure } & -3.44595 \mathrm{E}-04 \\ \text { metal loading*pressure } & 2.50000 \mathrm{E}-07\end{array}$

The LTCC firing shrinkage can be summarized as

follows: $\quad \%$ firing shrinkage $=13.6269$ -

(.00502500 x \%metal loading) - (.000344595 x pressure)

The contour plot of the shrinkage versus lamination pressure and metal loading is shown in Figure 1.

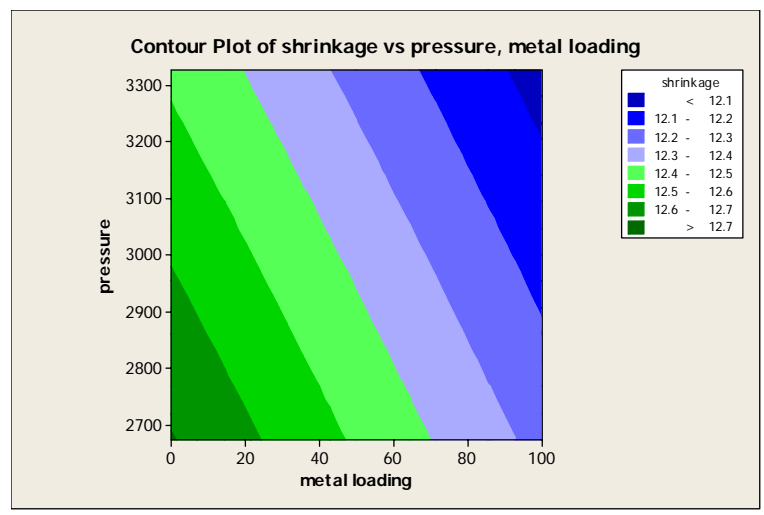

Figure 1

Based on the ANOVA, the factors "metal loading" and "lamination pressure" were identified as significant in terms of LTCC firing shrinkage. The contour plot defines the percent firing shrinkage for given inputs of metal loading and lamination pressure.

\section{DuPont 6.5 Mil Thick 951 P2 Green Tape}

The experimental set-up was replicated on the $6.5 \mathrm{mil}$ thick green tape (P2). The DOE run combinations and results following randomization are shown in Table 3.
Table 3

\begin{tabular}{|c|c|c|c|c|}
\hline Run Order & metal loading & pressure & layer count & shrinkage \\
\hline 1 & 25 & 3200 & 17 & 12.55 \\
\hline 2 & 50 & 3000 & 12 & 12.59 \\
\hline 3 & 50 & 3000 & 12 & 12.51 \\
\hline 4 & 50 & 3000 & 12 & 12.57 \\
\hline 5 & 25 & 2800 & 17 & 12.71 \\
\hline 6 & 75 & 3200 & 7 & 12.29 \\
\hline 7 & 50 & 3000 & 12 & 12.64 \\
\hline 8 & 25 & 2800 & 7 & 12.71 \\
\hline 9 & 75 & 3200 & 17 & 12.29 \\
\hline 10 & 25 & 3200 & 7 & 12.60 \\
\hline 11 & 75 & 2800 & 17 & 12.40 \\
\hline 12 & 75 & 2800 & 7 & 12.44 \\
\hline 13 & 50 & 2673 & 12 & 12.62 \\
\hline 14 & 0 & 3000 & 12 & 12.77 \\
\hline 15 & 50 & 3000 & 12 & 12.54 \\
\hline 16 & 50 & 3000 & 12 & 12.57 \\
\hline 17 & 50 & 3327 & 12 & 12.48 \\
\hline 18 & 50 & 3000 & 4 & 12.49 \\
\hline 19 & 100 & 3000 & 12 & 12.30 \\
\hline 20 & 50 & 3000 & 20 & 12.57 \\
\hline
\end{tabular}

The analysis of variance for the P2 tape can be summarized as shown in Table 4.

Table 4

\section{Response Surface Regression: shrinkage versus Block, metal loading, pressure}

Estimated Regression Coefficients for shrinkage P2

$\begin{array}{llcrc}\text { Term } & \text { Coef } & \text { SE Coef } & \text { T } & \text { P } \\ \text { Constant } & 12.5338 & 0.01122 & 1116.894 & 0.000 \\ \text { Block } & -0.0087 & 0.01122 & -0.780 & 0.448 \\ \text { metal loading } & -0.1620 & 0.01524 & -10.626 & 0.000 \\ \text { pressure } & -0.0569 & 0.01347 & -4.225 & 0.001 \\ \text { metal *pressure } & 0.0015 & 0.02156 & 0.072 & 0.944 \\ & & & \\ \text { S }=0.04917 \quad \text { R-Sq }=89.8 \% & \text { R-Sq(adj) }=87.0 \% & & \end{array}$

Estimated Regression Coefficients for shrinkage P2 using data in uncoded units

$\begin{array}{ll}\text { Term } & \text { Coef } \\ \text { Constant } & 13.6859 \\ \text { Block } & -0.00875000 \\ \text { metal loading } & -0.00597500 \\ \text { pressure } & -2.96982 \mathrm{E}-04 \\ \text { metal loading*pressure } & 2.50000 \mathrm{E}-07\end{array}$

The LTCC firing shrinkage can be summarized as follows: $\%$ shrikage $=\mathbf{1 3 . 6 8 5 9}$ (.00597500 x \% metal loading) - (.000296982 x pressure) 
The contour plot of the shrinkage versus metal loading, lamination pressure and layer count is shown in Figure 2.

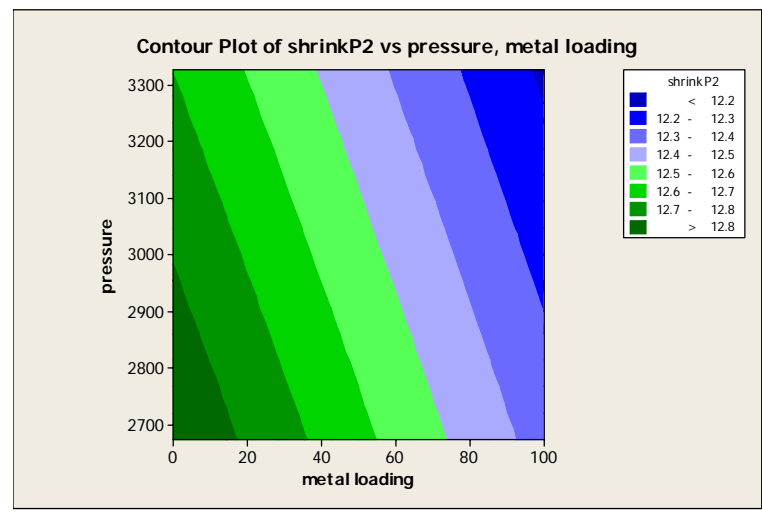

Figure 2

\section{DuPont 4.5 Mil Thick 951 PT Green Tape}

The experimental set-up was also replicated on the 4.5 mil thick green tape (PT). The DOE run combinations and results following randomization are shown in Table 5.

Table 5

\begin{tabular}{|ccccc|}
\hline Run Order & metal loading & pressure & layer count & shrinkage \\
1 & 25 & 3200 & 17 & 12.64 \\
2 & 50 & 3000 & 12 & 12.58 \\
3 & 50 & 3000 & 12 & 12.46 \\
4 & 50 & 3000 & 12 & 12.57 \\
5 & 25 & 2800 & 17 & 12.76 \\
6 & 75 & 3200 & 7 & 12.17 \\
7 & 50 & 3000 & 12 & 12.42 \\
8 & 25 & 2800 & 7 & 12.83 \\
9 & 75 & 3200 & 17 & 12.14 \\
10 & 25 & 3200 & 7 & 12.68 \\
11 & 75 & 2800 & 17 & 12.21 \\
12 & 75 & 2800 & 7 & 12.28 \\
13 & 50 & 2673 & 12 & 12.57 \\
14 & 0 & 3000 & 12 & 13.00 \\
15 & 50 & 3000 & 12 & 12.74 \\
16 & 50 & 3000 & 12 & 12.59 \\
17 & 50 & 3327 & 12 & 12.46 \\
18 & 50 & 3000 & 4 & 12.68 \\
19 & 100 & 3000 & 12 & 12.00 \\
20 & 50 & 3000 & 20 & 12.48 \\
\hline
\end{tabular}

The analysis of variance for the PT tape can be summarized as shown in Table 6.
Table 6

\section{Response Surface Regression: shrinkage versus Block, metal loading, pressure}

Estimated Regression Coefficients for PT shrinkage

$\begin{array}{lcccc}\text { Term } & \text { Coef } & \text { SE Coef } & \text { T } & \text { P } \\ \text { Constant } & 12.5217 & 0.01812 & 691.056 & 0.000 \\ \text { Block } & -0.0433 & 0.01812 & -2.392 & 0.034 \\ \text { metal loading } & -0.3185 & 0.02461 & -12.941 & 0.000 \\ \text { pressure } & -0.0472 & 0.02174 & -2.172 & 0.051 \\ \text { layer count } & -0.0402 & 0.02174 & -1.851 & 0.089 \\ \text { metal *pressure } & 0.0140 & 0.03481 & 0.401 & 0.696 \\ \text { metal *layer ct } & 0.0016 & 0.03481 & 0.045 & 0.965 \\ \text { pressure*layer ct } & 0.0087 & 0.02807 & 0.312 & 0.761\end{array}$

$\mathrm{S}=0.07940 \quad \mathrm{R}-\mathrm{Sq}=93.8 \% \quad \mathrm{R}-\mathrm{Sq}(\mathrm{adj})=90.2 \%$

Estimated Regression Coefficients for PT shrinkage using data in uncoded units

$\begin{array}{ll}\text { Term } & \text { Coef } \\ \text { Constant } & 14.4988 \\ \text { Block } & -0.0433333 \\ \text { metal loading } & -0.0171450 \\ \text { pressure } & -4.53610 \mathrm{E}-04 \\ \text { layer count } & -0.0347990\end{array}$

The LTCC firing shrinkage can be summarized as follows: \% firing shrinkage = 14.4988 (.0171450 x \% metal loading) - (.00045361 x pressure) - (.0347990 $x$ layer count)

The contour plots of the shrinkage versus metal loading, layer count, and lamination pressure are shown in Figure 3.

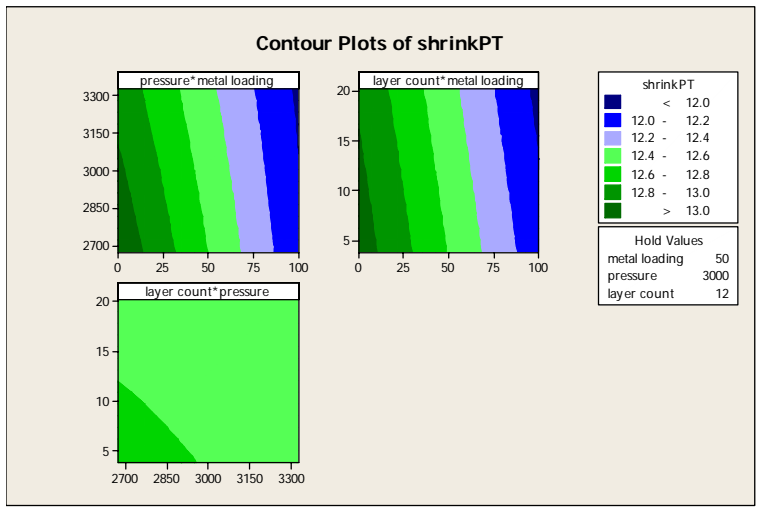

Figure 3

\section{Confirmation Run on RF MCMs (Metal Loading \& Expansion Factor Calculations):}

From the shrinkage prediction equations, a green tape expansion factor can be determined which is used for NC file generation as well as stencil and co-fired print screen fabrication. The green design expansion factor was determined as follows:

Green Design Expansion Factor = 1/(1-Predicted Shrinkage/100) 
In an effort to validate the equations, three subsequent products were fabricated using manufacturing outputs generated with these equations as shown in Tables 7, 8, and 9:

Table 7

\begin{tabular}{|c|c|c|c|}
\hline \multicolumn{4}{|l|}{ Product 1} \\
\hline \multirow[t]{6}{*}{ Gerber ( } & Tape Layer & Area & Metal Loading \\
\hline & & (Sq. In.) & (\%) \\
\hline & 1 & 0 & 0 \\
\hline & 2 & 0 & 0 \\
\hline & 3 & 0 & 0 \\
\hline & 4 & 0 & 0 \\
\hline Metal 01 & 5 & 0.0575 & 1.989619377 \\
\hline Metal 02 & 6 & 0.1147 & 3.968858131 \\
\hline Metal 03 & 7 & 0.7164 & 24.78892734 \\
\hline Metal 04 & 8 & 0.2441 & 8.446366782 \\
\hline Metal 05 & 9 & 0.2538 & 8.78200692 \\
\hline Metal 06 & 10 & 0.7201 & 24.91695502 \\
\hline Metal NS & 11 & 0.2252 & 7.792387543 \\
\hline Average & & 0.211981818 & 7.33501101 \\
\hline \multicolumn{4}{|c|}{$\begin{array}{l}\text { Note: Metal Loading Based on LTCC Network Area of 1.70" x 1.70" } \\
\text { (2.89 Sq. In.) }\end{array}$} \\
\hline \multicolumn{4}{|c|}{ Predicted Shrinkage Based on Average Metal Loading } \\
\hline \multicolumn{4}{|l|}{12.5562566} \\
\hline \multicolumn{4}{|c|}{$\begin{array}{l}\text { Calculated Expansion Factor for LTCC } 1 \text { Based on Averaqe Metal } \\
\text { Loading }\end{array}$} \\
\hline \multicolumn{4}{|l|}{1.1435924} \\
\hline \multicolumn{4}{|c|}{ Calculated Shrinkage Verification Dimension (Green State) } \\
\hline 5.71796198 & & & \\
\hline
\end{tabular}

The fired shrinkage verification dimension averaged 4.9997 inches compared to a nominal of 5.000 inches with a standard deviation of .0024 inch. The average shrinkage calculated for the lot of LTCC networks was $12.54 \%$ compared to the predicted shrinkage of $12.55 \%$. Product fabrication utilized progressive lamination with an initial pressure of 1200 psi and a final lamination pressure of 2750 psi.
Table 8

\begin{tabular}{|lccc|}
\hline $\begin{array}{l}\text { Product 2 } \\
\text { Gerber }\end{array}$ & Tape Layer & $\begin{array}{c}\text { Area } \\
\text { (Sq. In.) }\end{array}$ & $\begin{array}{c}\text { Metal Loading } \\
\text { (\%) }\end{array}$ \\
M00 & & 0.9027 & 56.59561129 \\
N00 & 1 & 0.0492 & 3.084639498 \\
P00 & 2 & 0.0435 & 2.727272727 \\
R00 & 3 & 0.9048 & 56.72727273 \\
Metal 05 & 4 & 0 & 0 \\
S00 & 5 & 0.7587 & 47.56739812 \\
T00 & 6 & 0.2075 & 13.00940439 \\
U00 & 7 & 1.0026 & 62.85893417 \\
V00 & 8 & 0.3492 & 21.89341693 \\
W00 & 9 & 0.3221 & 20.19435737 \\
Y00 & 10 & 1.0037 & 62.92789969 \\
Z00 & 11 & 0.24 & 15.04702194 \\
\multicolumn{4}{c}{} \\
Average & 12 & 0.482 & 30.21943574 \\
Note: Metal Loading Based on LTCC Network Area of 1.1" x 1.45" \\
(1.595 Sq. In.)
\end{tabular}

The fired shrinkage verification dimension averaged 4.999 inches compared to a nominal of 5.000 inches with a standard deviation of .0036 inch. The average shrinkage calculated for the lot of LTCC networks was $12.43 \%$ compared to the predicted shrinkage of $12.44 \%$. Product fabrication utilized progressive lamination with an initial pressure of 1200 psi and a final lamination pressure of 3000 psi. 
Table 9

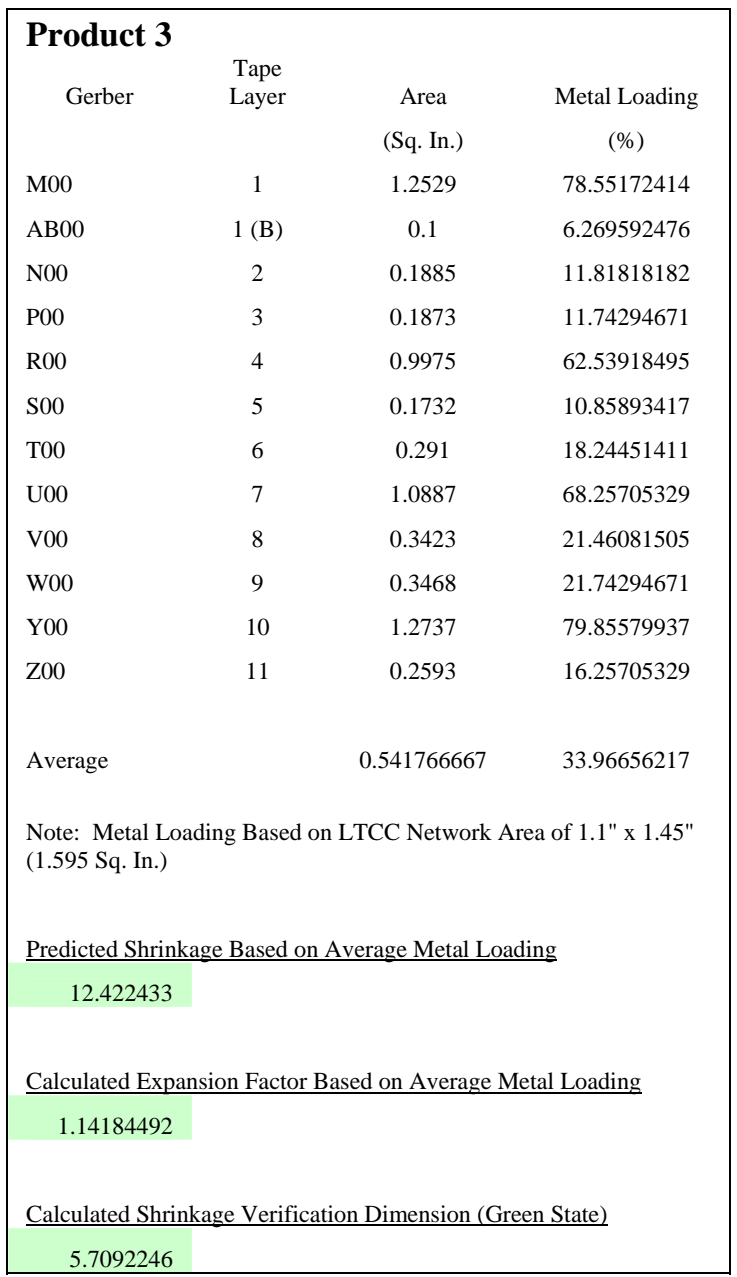

The fired shrinkage verification dimension averaged 4.9998 inches compared to a nominal of 5.000 inches with a standard deviation of .0036 inch. The average shrinkage calculated for the lot of LTCC networks was $12.43 \%$ compared to the predicted shrinkage of $12.42 \%$. Product fabrication utilized progressive lamination with an initial pressure of 1200 psi and a final lamination pressure of 2500 psi.
A typical RF MCM similar to the products validated in the confirmation run is shown in Figure 4.

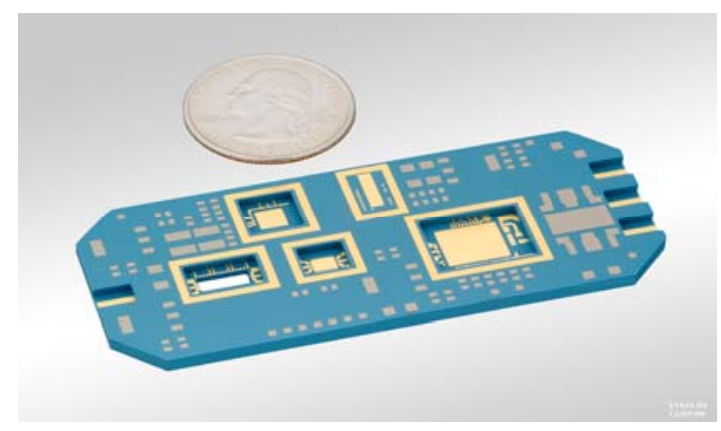

Figure 4

\section{Conclusions:}

The goal of the study was analyze and model the shrinkage behavior of DuPont 951 tape. Response Surface Analysis yielded a methodology to more accurately predict shrinkage of LTCC products based on design factors such as metal loading, layer count and tape thickness. This allowed for the adjustment of manufacturing outputs (punch files and cofired print tooling) to provide a final adjustment of shrinkage using lamination pressure. As the green tape thickness decreases the DOE factors and levels tested more closely models the shrinkage behavior as determined by the ANOVA. The accuracy of the shrinkage models was validated in the confirmation run builds of functional product.

The shrinkage models developed here apply to our specific process and equipment and it is highly recommended these experiments be repeated when applied to a different set of conditions.

\section{References:}

[1] Andreas Roosen, Dirk Pohle, Matthias Wagner, "Characterization of the Shrinkage Behavior of Pure, Screen Printed Thick Film Pastes and LTCC Green Tapes”, University of Erlangen - Nuremberg, Ceramic Interconnect Technology: The Next Generation II \& Tabletop Exhibition, April 26 - 28, 2004.

[2] Fournier, Y. , Bieri, L.S. , Maeder, T. , Ryser, P. , "Influence of lamination parameters on LTCC shrinkage under unconstrained sintering”, 4th European Microelectronics and Packaging Symposium - IMAPS, 2006, p. 165-170. 